\title{
Identification of Nonlinear Kinetics of Macroscopic Bio-reactions Using Multilinear Gaussian Processes ${ }^{\text {th }}$
}

\author{
Mingliang Wanga,b,*, Riccardo Sven Risuleo ${ }^{\mathrm{a}}$, Elling W. Jacobsen ${ }^{\mathrm{a}, \mathrm{b}}$, Véronique \\ Chotteau $^{\mathrm{b}, \mathrm{c}}$, Håkan Hjalmarsson ${ }^{\mathrm{a}, \mathrm{b}}$ \\ ${ }^{a}$ Division of Decision and Control Systems, KTH Royal Institute of Technology \\ ${ }^{b}$ Centre for Advanced Bioproduction, KTH Royal Institute of Technology \\ ${ }^{c}$ Department of Industrial Biotechnology, KTH Royal Institute of Technology
}

\begin{abstract}
In biological systems, nonlinear kinetic relationships between metabolites of interest are modeled for various purposes. Usually, little a priori knowledge is available in such models. Identifying the unknown kinetics is, therefore, a critical step which can be very challenging due to the problems of (i) model selection and (ii) nonlinear parameter estimation. In this paper, we aim to address these problems systematically in a framework based on multilinear Gaussian processes using a family of kernels tailored to typical behaviours of modulation effects such as activation and inhibition or combinations thereof. Using one such process as a model for each modulation effect leads to a much more flexible model than conventional parametric models, e.g., the Monod model. The resulting models of the modulation effects can also be used as a starting point for estimating parametric kinetic models. As each modulation effect is modeled separately, this task is greatly simplified compared to the conventional approach where the parameters in all modulation functions have to be estimated simultaneously. We also show how the type of modulation effect can be selected automatically by way of regularization, thus by-passing the model selection problem. The resulting parameter estimates can be used as initial estimates in the conventional approach where the full model is estimated. Numerical experiments, including fed-batch simulations, are conducted to demonstrate our methods.
\end{abstract}

Keywords: Gaussian process, model selection, parameter estimation, Monod model, kinetics, macroscopic modeling, nonlinear systems

\section{Introduction}

Macroscopic models are systems that describe the mass balance between macroscopic species, e.g. biomass, main substrates and products of interest. They play an essential role

\footnotetext{
${ }^{*}$ I am corresponding author

Email addresses: miwan@kth.se (Mingliang Wang), risuleo@kth.se (Riccardo Sven Risuleo), jacobsen@kth.se (Elling W. Jacobsen), veronique.chotteau@biotech.kth.se (Véronique Chotteau), hjalmars@kth.se (Håkan Hjalmarsson) 
in biological systems for the purpose of product prediction, media optimization and feeding control.

To build the macroscopic models, black-box models are popular in the literature as they require little prior information of the bio-system, e.g. artificial neural networks (Chen et al., 2000), Gaussian process (GP) models (Del Rio-Chanona et al., 2019; Bradford et al., 2018). Black models have satisfactory modeling performance after careful training with the experimental data (Grosfils et al., 2005). However, they are totally data-driven approaches with little biological interpretations, even though some of them are hybrid (Von Stosch et al., 2014; Fiedler and Schuppert, 2008), e.g. incorporating the law of mass balance (Oliveira, 2004; Wouwer et al., 2004). In addition, it is hard to specify the model complexity for black-box approach in advance. This will lead to under-fitting or over-fitting problems when there is a mismatch between the model complexity and data.

To build the biologically interpretable kinetics, the enzyme-catalytic effects or modulation effects are the key factors (Savageau, 1969a,b). Individual modulation effects such as activation, inhibitions, limitation are commonly described in rational structures, e.g. the Michaelis-Menten model (Menten and Michaelis, 1913). When little a priori knowledge of the biological systems is available, it is common to build the kinetics as a product of individual modulation phenomena (Batt and Kompala, 1989; Haag et al., 2005; Leskovac, 2003). The identification of the models requires (i) model selection and (ii) parameter estimation which both can be challenging due to nonlinear model structure (Haag et al., 2005).

A popular method for model selection is cross validation which selects the candidate models based on their predictive performance. Information criteria, e.g. the Akaike information criterion (AIC) (Akaike, 1998) and the Bayesian information criterion (BIC) (Schwarz and Others, 1978), are also in common use. Other methods like residual analysis (Pagan and Hal1, 1983), F-test (Hahs-Vaughn and Lomax, 2013), confidence region test (Aitchison, 1964) are also available but often restricted to linear models. It is possible to extend these methods to nonlinear models. In a recent paper (Lillacci and Khammash, 2010), the confidence region test is used to select nonlinear models by checking whether the estimate of variance is in the confidence region or not. Likelihood ratio test is also proposed to select the most likely kinetic structure among candidate models (Mailier and Wouwer, 2012). However, the number of model alternatives that needs to be examined generally grows combinatorically in the above methods and prevents these methods to be used in large-scale problems. This is exactly what happens in our case since the number of candidate models grows geometrically with the number of modulation effects (Mangan et al., 2016). With $d$ different types of modulation effects and $m$ different modulation effects taking place, the total number of candidate models will be $d^{m}$. Note that $m$ can be more than 20 in some large-scale biological systems (Hagrot et al., 2017). Certain regularization methods, e.g. LASSO (Zou, 2006; Tibshirani, 1996), are good alternatives as they select models by trading off between the model accuracy and complexity (Brunton et al., 2016) in a continuous fashion, avoiding the combinatorial complexity problem. An in-depth review of these methods can be found in (Ding et al., 2018). In addition, an interesting approach is proposed in a recent paper (Olofsson et al., 2019) using Gaussian processes as surrogate models for model discrimination.

General models that circumvent the model selection problems are also available for de- 
scribing individual modulation effect, e.g. Batt and Kompala (1989) use the Monod type kinetic expression as a general model. However, this will result in a nonlinear and overparameterized model with $2 m+1$ parameters. To address the problem, Haag et al. (2005) proposed a piecewise function as a general model for the inhibitory and limiting (saturation) effects which reduces the number of parameter to $m+1$. However, the model does not cover the non-competitive inhibition type kinetics.

In addition, parameter estimation in kinetics models is also difficult due to non-convexities arising from the non-linear nature of the Monod type of model. Direct parameter estimation, e.g. the nonlinear least-squares method (Marquardt, 1963; Toulias and Kitsos, 2016), and Newton-type methods (Heiser and Parrish, 1989; Dimitrov and Kamenski, 1991), can become challenging due to the issues of local minima and finding good initial estimates. For models that are rational in the parameters. One way to avoid local optima is to consider the equation error obtained by multiplying the model equation with the denominator and then reparametrize the model such that a linear in the parameters expression is obtained (Andrews, 1968). However, this results in an errors-in-variables (EIV) problem (Carroll et al., 2006) for which additional measures are required to ensure consistency (Wang et al., 2019). The reparametrization also leads to an overparametrization, potentially reducing the estimation accuracy. Instead of using the classical Monod type models, Bogaerts et al. (1999) developed an exponential model, later improved by Grosfils et al. (2007), for a systematic identification approach which allows to obtain maximum likelihood estimates of parameters after linearization. Following the same idea, Richelle and Bogaerts (2015) transform the identified exponential models to the classical Monod type models for better biological interpretations.

Considering the above facts, in this contribution we propose to address the model selection and parameter estimation problems in a systematic framework, which can be summarized in the following steps:

1. Nonparametric model estimation. Each individual modulation effect is modeled as a multilinear Gaussian process (GP) which is estimated from data.

2. Estimation of individual parametric models. The estimate of each modulation effect from the previous step is used as "data" in a regularization based identification scheme (RIS) to estimate a parametric model for that modulation effect. The regularization is used to automatically select the type of modulation effect each metabolite has.

3. Estimation of a complete kinetic model. The modulation functions selected in Step 2 are combined into a complete parametric model. Starting from the parameter values obtained from the previous step, the parameters of this model are fine-tuned minimizing the discrepancy between the model predictions and the data.

The details of the methods are provided in Section 2. The performance of the proposed algorithm is validated through simulations in Section 3, and Section 4 concludes the paper.

Notation. $\mathcal{N}(x ; m, \Sigma)$ denotes the probability density function of a Gaussian random variable with mean $m$ and covariance $\Sigma$. $\boldsymbol{z} \sim \mathcal{N}(m, \Sigma)$ denotes that the random variable $\boldsymbol{z}$ is 
Gaussian distributed with mean $m$ and covariance matrix $\Sigma . \mathrm{p}(x ; \theta)$ denotes a probability density function parametrized by the vector $\theta$.

\section{Methods}

We assume that the reaction rate $v$ of a macroscopic reaction can be modeled as a product of individual modulation effects from $m$ metabolites (Hagrot et al., 2017, 2019)

$$
v[t]=v_{\max } \prod_{i=1}^{m} h_{i}\left(c_{i}[t]\right),
$$

where $c_{i}[t]$ is the concentration of the $i_{\text {th }}$ metabolite at time $t$ and the function $h_{i}$ denotes the modulation effect, e.g. activation or inhibition, of the $i_{t h}$ metabolite. There are various parametric models for describing the modulation effects (Batt and Kompala, 1989), e.g. Monod, Tessier and Ming laws. Among them, the Monod-type model is the most popular and also the one used in this paper. However, it is easy to adapt the presented methods to other models as well. For a uniform mathematical treatment, we add an artificial modulation effect named "neutral" to denote that a metabolite has negligible or no effect on the reaction. In total we will consider four types of modulation functions

$$
\begin{aligned}
h_{a c t}\left(c ; k_{a c t}\right) & :=\frac{c}{c+k_{a c t}}, \\
h_{i n h}\left(c ; k_{i n h}\right) & :=\frac{1}{1+k_{i n h} c}, \\
h_{d c}\left(c ; k_{a c t}, k_{i n h}\right) & :=\frac{c}{c+k_{a c t}} \cdot \frac{1}{1+k_{i n h} c}, \\
h_{n o}(c) & :=1,
\end{aligned}
$$

where $c$ denotes the input, $k_{a c t}$ and $k_{i n h}$ are model parameters, the subscripts "act", "inh", " $d c$ ", "no" denote the effects of activation, inhibition, double-component and "neutral", respectively. Examples of these functions are shown in Figure 1.

In practice, even the type of modulation effect of a metabolite in macroscopic reactions is seldom known. This means that for each metabolite we have to select a proper $h_{i}$ from the four candidate functions (2)-(5) using the information contained in the experimental data. This is thus a model selection problem.

Considering the above facts, a three-step framework is proposed. In Step 1, a multilinear Gaussian process (GP) is used as a flexible non-parametric model to infer the modulation effect of each metabolite. Each modulation function $h_{i}$ is modelled as a zero-mean GP model where realizations of the GP represent candidates for the modulation effect of that particular metabolite. Furthermore, by letting the kernel be parametrized (by so called hyperparameters) and tuning these using data, these realizations can be adapted to the actual modulation effects. This tuning is done by maximizing the (marginal) likelihood of the data. Once the hyperparameters have been determined, we can compute the posterior 

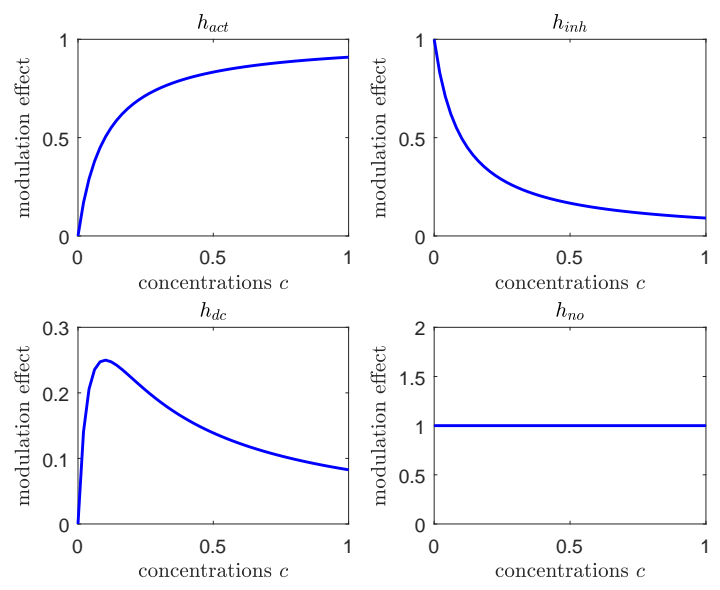

Figure 1: Modulation functions: activation (top-left) with $k_{a c t}=0.25$, inhibition (top-right) with $k_{i n h}=$ 0.25 , double-component (bottom-left) with $k_{a c t}=0.25, k_{i n h}=0.25$, neutral-component (bottom-right).

means of the GPs as estimates of the modulation effects. We elaborate in more detail on this in Section 2.1.

One benefit of this first step is that the GPs are able to adapt to the actual modulation effects. Furthermore, the individual estimates of the modulation effects can be used as "data" to estimate parametric models which means that the original large-scale model selection and estimation problem, where parametric models of all the $h_{i}$ in (1) are to be estimated simultaneously from data, is now reduced into $m$ independent and small-scale model selection and estimation problems. Step 2 builds on this notion. In Section 2.2 we develop a regularization based identification scheme for model selection. We start by observing that the double component function can be used to represent also the other three modulation functions:

$$
\begin{aligned}
& h_{d c}(c ; k, 0)=h_{a c t}(c ; k), \\
& h_{d c}(c ; 0, k)=h_{\text {inh }}(c ; k), \\
& h_{d c}(c ; 0,0)=h_{n o}(c)=1 .
\end{aligned}
$$

Therefore, in the second step we assume a double-component structure for each $h_{i}$ in the model (1). However, this model is over-parameterized unless the actual modulation effect is of double-component type. To address this problem, we develop a $\ell_{1}$-norm regularized identification scheme $\left(\ell_{1}\right.$-RIS) in which "trivial" parameters can be shrunk to 0 , effectively meaning that the important modulation effects are selected.

Finally, in Step 3, we perform classical parameter estimation of a full scale kinetics model. Here we benefit from the outcomes of Step 2 in two ways: i) We use the modulation effects resulting from the $\ell_{1}$-regularization, i.e. parameters deemed to be zero in that step are kept fixed. ii) The parameter estimates from Step 2 are used as initial values in the optimization. We discuss this step in Section 2.3. 


\subsection{Step 1 - Gaussian process modeling}

\subsubsection{Gaussian processes for regression}

In this section, we will give a brief introduction to the standard Gaussian process model and its non-parametric nature to convey better understanding of the proposed multilinear Gaussian process. Consider a general regression model

$$
y=f(\boldsymbol{x})+e,
$$

where $\boldsymbol{x} \in \mathcal{R}^{m}$ and $y \in \mathcal{R}$ are the input and output, $f$ is the unknown function, $e$ is a random variable representing measurement noise. For simplicity, we assume the noise for different observations $y$ to be independent and identically distributed (i.i.d) following a zero mean Gaussian distribution $e \sim \mathcal{N}\left(0, \sigma_{e}^{2}\right)$.

Given the observations $\boldsymbol{y}=\left\{y_{i}\right\}_{i=1}^{N}$, corresponding to given points $\left\{\boldsymbol{x}_{i}\right\}_{i=1}^{N}$, the objective is to estimate the unknown function $f$. A Gaussian process model defines a Gaussian distribution over $f$ (Rasmussen, 2004). The distribution of $f$ is characterized by the mean function $\mu(\boldsymbol{x})$ and covariance function (or kernel) $K\left(\boldsymbol{x}, \boldsymbol{x}^{\prime}\right)$

$$
\begin{aligned}
\mu(\boldsymbol{x}) & =\mathbb{E}[f(\boldsymbol{x})], \\
K\left(\boldsymbol{x}, \boldsymbol{x}^{\prime}\right) & =\mathbb{E}\left[(f(\boldsymbol{x})-\mu(\boldsymbol{x}))\left(f\left(\boldsymbol{x}^{\prime}\right)-\mu\left(\boldsymbol{x}^{\prime}\right)\right)\right],
\end{aligned}
$$

where $\mu(\boldsymbol{x})$ is often assumed to be 0 . The kernel $K\left(\boldsymbol{x}, \boldsymbol{x}^{\prime}\right)$ can be parametrized by a set of hyperparameters $\theta$. These parameters are used to tailor the kernel to the actual function that is estimated. For example, a commonly used kernel is the squared exponential (SE) kernel,

$$
K_{\theta}\left(\boldsymbol{x}, \boldsymbol{x}^{\prime}\right)=\sigma_{s e} \exp \left(-\frac{\left\|\boldsymbol{x}-\boldsymbol{x}^{\prime}\right\|^{2}}{2 \ell^{2}}\right)
$$

where $\theta=\left\{\sigma_{s e}, \ell\right\}$.

By tuning the hyperparameters, we can tune the whole family of candidate functions $f$ as shown in Figure 2 (a) and (b) which depicts five realizations of $f$ for two different sets of hyperparameters. The more representative the realizations are of the actual function that we would like to model, the more accurate the model will be. A common approach to achieve this is to take the $\theta$ that maximizes the likelihood of the given observations $\boldsymbol{y}$, i.e.

$$
\theta_{\star}=\underset{\theta}{\arg \max } \log \mathrm{p}(\boldsymbol{y} ; \theta) .
$$

This method to determine hyperparameters is known as Empirical Bayes (Casella, 1985). Since a closed form expression of $\log \mathrm{p}(\boldsymbol{y} ; \theta)$ is available for a GP, it is straightforward to use numerical optimization to solve (11). Using the kernel $K_{\theta_{\star}}$ and the observations $\boldsymbol{y}$, we subsequently update the prior distribution to a posterior distribution $\mathrm{p}\left(f \mid \boldsymbol{y} ; \theta_{\star}\right)$. An example for the posterior distribution is presented in Figure 2c, where the posterior mean in blue line is often used as the estimate of $f$. The details of the likelihood $\mathrm{p}(\boldsymbol{y} ; \theta)$, together with the prior and posterior distributions, are elaborated in Appendix A. 

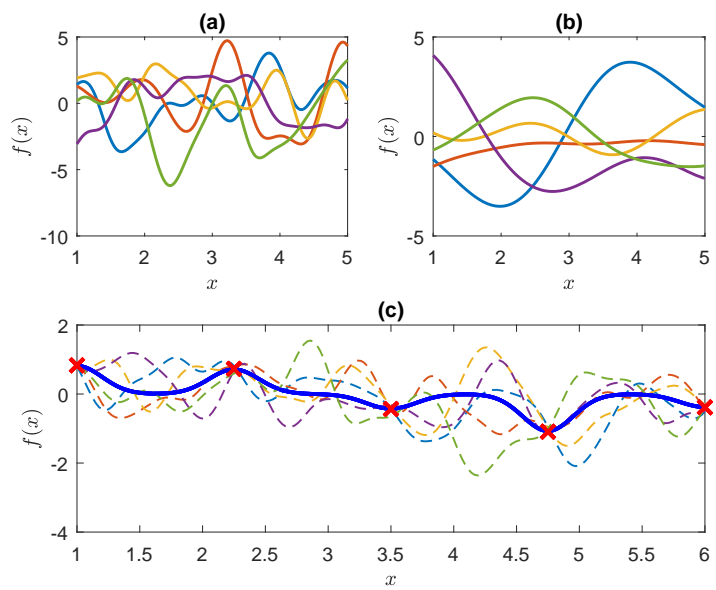

Figure 2: (a) Five realizations of the prior distribution $\mathcal{N}\left(0, K_{\theta}(\boldsymbol{x}, \boldsymbol{x})^{\prime}\right)$ with $\theta=\{2,0.3\}$; (b) Five realizations of the prior distribution with $\theta=\{2,1\}$; (c) Five realizations and the mean of the posterior distribution $p(f \mid \boldsymbol{y})$; the observations are in red $\times$, the blue line is the posterior mean.

\subsubsection{Multilinear Gaussian processes}

In this part, we will illustrate the idea of using a multilinear Gaussian process in the model (1). For convenience of presentation, we illustrate the method using a bilinear kinetic model

$$
v[t]=\gamma_{1} h_{1}\left(c_{1}[t], k_{1}\right) \gamma_{2} h_{2}\left(c_{2}[t], k_{2}\right)
$$

where $c_{1}$ and $c_{2}$ are the concentrations of two metabolites, where $\gamma_{1} \gamma_{2}=v_{\max }$ and where $h_{1}$ and $h_{2}$ are the corresponding modulation functions to be determined. We assume that we have measurements $y[t]$ of reaction rate $v[t]$ that are contaminated by independent additive Gaussian noise $e[t] \sim N\left(0, \sigma_{e}^{2}\right)$ :

$$
y[t]=v[t]+e[t]
$$

We neglect measurement noise in the concentrations. In this paper, we introduce the kernel function

$$
\begin{aligned}
K_{\theta}\left(x, x^{\prime}\right) & =\theta_{1} \exp \left[-\theta_{2}\left(\log (x)-\log \left(x^{\prime}\right)\right)^{2}\right] \\
& =\theta_{1}\left(x / x^{\prime}\right)^{-\theta_{2} \log \left(x / x^{\prime}\right)}
\end{aligned}
$$

for all types of modulation effects, where $\theta_{1}, \theta_{2}$ are the hyperparameters. To see the behavior of this kernel, we show an example in Figure 3 which demonstrates that the kernel is able to capture the dynamics of all 4 types of modulation effects. Then, for (12), we introduce the model

$$
v[t]=f[t] \cdot g[t]
$$



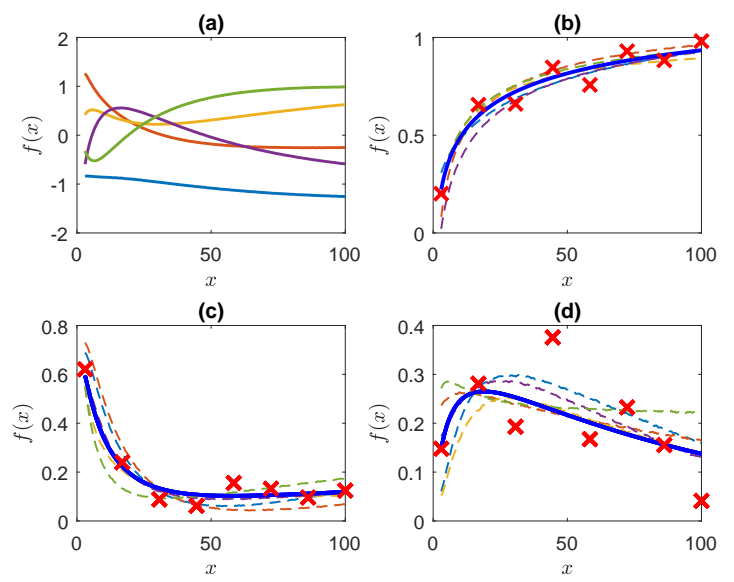

Figure 3: Performance of the kernel (14): (a) 5 samples of $f \sim K_{\vartheta}\left(x, x^{\prime}\right)$ with $\vartheta=\{0.5,0.2\}$; (b) the dashed lines are samples of posterior distribution of $h_{a c t}$, the red $\times$ are the data, the blue line is the posterior mean; (c) posterior distribution of $h_{i n h}$; (d) posterior distribution of $h_{d c}$.

where $f$ and $g$ are modeled as independent Gaussian processes,

$$
f \sim \mathcal{N}\left(0, K_{\alpha}\left(c_{1}, c_{1}^{\prime}\right)\right), \quad g \sim \mathcal{N}\left(0, K_{\beta}\left(c_{2}, c_{2}^{\prime}\right)\right)
$$

and $K_{\alpha}$ and $K_{\beta}$ are covariance functions in (14) with $\alpha, \beta$ being the hyperparameters, $c_{1}, c_{1}^{\prime}$ and $c_{2}, c_{2}^{\prime}$ are concentrations of metabolite 1 and 2 , respectively.

Now we have the model input $\left\{c_{1}[t], c_{2}[t]\right\}_{t=1}^{N}$ and output $\boldsymbol{y}=\{y[t]\}_{t=1}^{N}$ as our data set. We consider $\boldsymbol{f}=\{f[t]\}_{t=1}^{N}, \boldsymbol{g}=\{g[t]\}_{t=1}^{N}$ as latent variables since they are indirectly observed through $\boldsymbol{y}$. In the following, we will suppress the model input in the notation.

As in the case of one GP, cf. (11), the unknown parameters

$$
\Theta=\left\{\alpha, \beta, \sigma_{e}^{2}\right\}
$$

are estimated by maximizing the log (marginal) likelihood,

$$
L(\Theta)=\log \mathrm{p}(\boldsymbol{y} ; \Theta),
$$

However, unlike the situation in Section 2.1.1, the marginal likelihood in (18) is non-Gaussian due to the product of two Gaussian random variables in (15). This makes it difficult to compute a closed form expression for $\mathrm{p}(\boldsymbol{y} ; \Theta)$. To counter this we take a sampling approach where instead of computing the required distributions we generate samples from them and use these to approximate the quantities required to compute the hyperparameters and the posterior mean. More precisely, we combine the expectation maximization (EM) algorithm for maximum likelihood estimation (Dempster et al., 1977; McLachlan and Krishnan, 2007) with Gibbs sampling (Casella and George, 1992). 
The EM algorithm is defined as iterating the following two steps

$$
\begin{aligned}
\text { "E-step" } \quad Q_{n}(\Theta) & =\iint \log \mathrm{p}(\boldsymbol{y}, \boldsymbol{g}, \boldsymbol{f} ; \Theta) \mathrm{p}\left(\boldsymbol{g}, \boldsymbol{f} \mid \boldsymbol{y} ; \Theta_{n}\right) d \boldsymbol{f} d \boldsymbol{g} \\
\text { "M-step" } \quad \Theta_{n+1} & =\underset{\alpha, \beta}{\arg \max } Q_{n}(\Theta)
\end{aligned}
$$

where $n$ is the iteration step, $\Theta_{n}$ is the parameter obtained at the $n_{\text {th }}$ step. The EM algorithm is known to produce a sequence $\left\{\Theta_{n}\right\}$ that monotonically increases the (marginal) likelihood function, converging to a local optimum $\Theta_{\star}$ of this function. Occasionally, convergence to saddle-points or even local minima may occur but reports of such cases are rare.

We can rewrite the joint distribution in the "E step" as

$$
\mathrm{p}(\boldsymbol{y}, \boldsymbol{g}, \boldsymbol{f} ; \Theta)=\mathrm{p}\left(\boldsymbol{y} \mid \boldsymbol{g}, \boldsymbol{f} ; \sigma_{e}^{2}\right) \mathrm{p}(\boldsymbol{g}, \boldsymbol{f} ; \alpha, \beta)
$$

Then, the "E step" in (19) can be rewritten as

$$
Q_{n}(\Theta)=\iint\left[\log \mathrm{p}\left(\boldsymbol{y} \mid \boldsymbol{g}, \boldsymbol{f} ; \sigma_{e}^{2}\right)+\log \mathrm{p}(\boldsymbol{g}, \boldsymbol{f} ; \alpha, \beta)\right] \mathrm{p}\left(\boldsymbol{g}, \boldsymbol{f} \mid \boldsymbol{y} ; \Theta_{n}\right) d \boldsymbol{f} d \boldsymbol{g}
$$

Since the posterior $\mathrm{p}\left(\boldsymbol{g}, \boldsymbol{f} \mid \boldsymbol{y} ; \Theta_{n}\right)$ is not available in closed form, we use the Monte Carlo method to approximate (22),

$$
\hat{Q}_{n, M}(\Theta):=\frac{1}{M} \sum_{j=1}^{M} \log \mathrm{p}\left(\boldsymbol{y} \mid \boldsymbol{f}_{j}, \boldsymbol{g}_{j} ; \sigma_{e}^{2}\right)+\frac{1}{M} \sum_{j=1}^{M} \log \mathrm{p}\left(\boldsymbol{f}_{j} ; \alpha\right)+\frac{1}{M} \sum_{j=1}^{M} \log \mathrm{p}\left(\boldsymbol{g}_{j} ; \beta\right),
$$

where $\left\{\boldsymbol{f}_{j}, \boldsymbol{g}_{j}\right\}_{j=1}^{M}$ are samples drawn from $\mathrm{p}\left(\boldsymbol{g}, \boldsymbol{f} \mid \boldsymbol{y} ; \Theta_{n}\right)$. As already pointed out, $\mathrm{p}\left(\boldsymbol{g}, \boldsymbol{f} \mid \boldsymbol{y} ; \Theta_{n}\right)$ is not easily computable and therefore it is not straightforward to generate samples from this distribution either. However, the conditional distributions $\mathrm{p}(\boldsymbol{g} \mid \boldsymbol{f}, \boldsymbol{y} ; \beta)$ and $\mathrm{p}(\boldsymbol{f} \mid \boldsymbol{g}, \boldsymbol{y} ; \alpha)$ are Gaussian

$$
\begin{aligned}
\mathrm{p}(\boldsymbol{g} \mid \boldsymbol{f}, \boldsymbol{y} ; \beta) & =\mathcal{N}\left(\boldsymbol{m}_{g}, \boldsymbol{P}_{g}\right), \\
\mathrm{p}(\boldsymbol{f} \mid \boldsymbol{g}, \boldsymbol{y} ; \alpha) & =\mathcal{N}\left(\boldsymbol{m}_{f}, \boldsymbol{P}_{f}\right),
\end{aligned}
$$

where

$$
\begin{aligned}
\boldsymbol{P}_{g}^{-1} & =\frac{1}{\sigma_{e}^{2}} \boldsymbol{D}_{f}^{2}+\boldsymbol{K}_{\beta}^{-1}, \boldsymbol{m}_{g}=\frac{1}{\sigma_{e}^{2}} \boldsymbol{P}_{g} \boldsymbol{D}_{f} \boldsymbol{y} \\
\boldsymbol{P}_{f}^{-1} & =\frac{1}{\sigma_{e}^{2}} \boldsymbol{D}_{g}^{2}+\boldsymbol{K}_{\alpha}^{-1}, \boldsymbol{m}_{f}=\frac{1}{\sigma_{e}^{2}} \boldsymbol{P}_{f} \boldsymbol{D}_{g} \boldsymbol{y}
\end{aligned}
$$

Here $\boldsymbol{D}_{f}=\operatorname{diag}(\boldsymbol{f})$ and $\boldsymbol{K}_{\beta}$ is the Gram matrix with respect to kernel function $K_{\beta}$. We can therefore instead use what is known as Gibbs sampling, alternating between drawing samples from (24) and (25). This forms a Markov Chain with the desired p $\left(\boldsymbol{g}, \boldsymbol{f} \mid \boldsymbol{y} ; \Theta_{n}\right)$ as stationary distribution. The implementations of Gibbs sampling and the EM algorithm are given in Appendix B. 
As estimates of $\gamma_{1} h_{1}[t]$ and $\gamma_{2} h_{2}[t]$ in (14) we will use approximations of the corresponding posterior means of our multilinear GP model. To this end, we use Gibbs sampling again to take samples of the posterior distribution $\mathrm{p}\left(\boldsymbol{g}, \boldsymbol{f} \mid \boldsymbol{y} ; \Theta_{\star}\right)$. With the samples from the Gibbs sampling denoted as $\left\{\boldsymbol{f}_{l}^{\star}\right\}_{l=1}^{M}$ and $\left\{\boldsymbol{g}_{l}^{\star}\right\}_{l=1}^{M}$, the posterior mean approximations are given by the sample means

$$
\hat{h}_{1}[t]=\frac{1}{M} \sum_{l=1}^{M} \boldsymbol{f}_{l}^{\star}[t], \quad \hat{h}_{2}[t]=\frac{1}{M} \sum_{l=1}^{M} \boldsymbol{g}_{l}^{\star}[t],
$$

and the estimated output is taken as

$$
\hat{v}[t]=\hat{h}_{i}[t] \cdot \hat{h}_{j}[t] .
$$

We have illustrated the method for the bilinear case but it extends readily to an arbitrary number of metabolites, though the sampling becomes more and more intense as the number of metabolites increases.

\subsection{Step 2-Estimation of Monod kinetics for individual kinetic components}

The multilinear GP approach in Section 2.1 gives estimates $\hat{h}_{i}$ for each metabolite, $i=$ $1, \ldots, m$. It is now easy to fit one Monod-type model to each one of these using a quadratic cost function

$$
\begin{gathered}
J_{i}\left(k_{a c t}, k_{i n h}, \gamma\right):=\sum_{t=1}^{N}\left[\hat{h}_{i}[t]-\gamma h_{d c}\left(c_{i}[t] ; k_{a c t}, k_{i n h}\right)\right]^{2}, \\
\text { s.t. } \quad k_{a c t} \geq 0, k_{\text {inh }} \geq 0, \gamma \geq 0
\end{gathered}
$$

where $\hat{h}_{i}[t]$ is the approximation of the posterior mean as in (27) at time $t$, and $\gamma$ is the scaling parameter as introduced in (12). To distinguish between the cases of activation, inhibition, double-component and neutral which corresponds to different combinations of $\left\{k_{a c t}, k_{i n h}\right\}$ where either 0,1 or 2 of these coefficients are zero, see (6), we can use so called $\ell_{1}$-regularization where a penalty term proportional to the sum of the magnitudes of the parameters is added to the criterion. Adding such a term to (29), parameters that are small when (29) is minimized, are shrunk to become identically zero (Schmidt, 2005; Donoho, 2006; Park and Hastie, 2007). The resulting criterion is

$$
J_{i}\left(k_{a c t}, k_{i n h}, \gamma\right)+\lambda\left(k_{a c t}+k_{i n h}\right),
$$

where $\lambda$ is a penalty factor that represents the trade off between the loss function $J$ and the $\ell_{1}$-norm regularization terms (since $k_{a c t}, k_{i n h}$ are both positive values, the absolute value is neglected). The parameters, identified by minimizing (30), are denoted as $\left\{\hat{k}_{i, a c t}, \hat{k}_{i, i n h}, \hat{\gamma}_{i}\right\}_{i=1}^{m}$. K-fold cross validation (Kohavi and Others, 1995) is a common way to choose $\lambda$ such that it is as large as possible (meaning as many parameters as possible are shrunk to zero) while still maintaining a good fit of the Monod model to the Gaussian process model. Limitations in numerical precision can cause the resulting estimates $\hat{k}_{i, a c t}$ and $\hat{k}_{i, i n h}$ to be non-zero but small. Therefore it is common to force the estimates to be zero if they are smaller than some small $\epsilon$. The end result is a model which belongs to one of the four models (2)-(5). 


\subsection{Step 3 - Fine-tuning of parameters}

In Step 3, the identified modulation functions from Step 2 are combined into one model,

$$
\hat{v}[t]=v_{\max } \prod_{i=1}^{m} h_{d c}\left(c_{i}[t] ; k_{i, a c t}, k_{i, i n h}\right) .
$$

The parameters in (31) are then fine-tuned using the nonlinear least squares method (NLLS) (Strutz, 2010) to minimize the cost function $\sum_{t=1}^{N}(y[t]-\hat{v}[t])^{2}$. Parameters $k_{i, a c t}$ and $k_{i, i n h}$ that were estimated to be zero in Step 2 are kept fixed which effectively means that we use the type of modulation effects that were determined in this step. The initial values are important for NLLS to avoid the local minima and here we benefit from Step 2 by using the estimates from this step as initial values and $\prod_{=1}^{m} \hat{\gamma}_{i}$ as initial value for $v_{\max }$.

\subsection{Comments on identifiability}

Identifiability concerns the notion of being able to distinguish one model from another (Lennart, 1999). In our context, this concerns the ability to separate the two functions $f$ and $g$ in (15) from measurements of their product.

For a parametric model this depends on the parameterization of the models. Suppose that $f$ is an activation function

$$
f[t]=\frac{c_{1}[t]}{c_{1}[t]+k_{1}}
$$

and $g$ an inhibition function

$$
g[t]=\frac{1}{1+k_{2} c_{2}[t]} .
$$

Then for given product consistent with these functions

$$
h\left(c_{1}, c_{2}\right):=v_{\max } \frac{c_{1}}{\left(c_{1}+k_{1}^{\prime}\right)\left(1+k_{2}^{\prime} c_{2}\right)},
$$

$v_{\max }, f$ and $g$ can be uniquely determined due to the restrictions imposed by the parameterization on the two functions. However, when only measurements of the product are available

$$
v[t]=h\left(c_{1}[t], c_{2}[t]\right) .
$$

This depends also on the concentrations $c_{1}[t]$ and $c_{2}[t]$, i.e. the input excitation. If $c_{1}[t]=$ $c_{2}[t]=1, t=1,2,3, \ldots$, then clearly for any given $k_{1}$ one can find $v_{\max }$ and $k_{2}$ such that the product $f[t] g[t]$ is consistent with the data. However, when the inputs are different, the functional restrictions allow the functions to be separated.

Now, when Gaussian process models are used for the two functions it may seem impossible to separate the two functions regardless of the input excitation as such models in principle allow any shape of the functions. Hence, for any given $f[t], t=1,2,3, \ldots$, one could take $g[t]=v[t] / f[t]$. However, also here the functions are in a sense restricted albeit in a less obvious manner. This is the purpose of the prior which introduces an ordering of the 
candidate functions which is reflected by the a priori probabilities $\mathrm{p}(f)$ and $\mathrm{p}(g)$. To appreciate the mechanism, recall that the estimate of one of the functions is its posterior mean, which is nothing but the integral over all models weighted with their posterior probabilities. Now the posterior probability of a given pair $(f, g)$ is given by the formula

$$
p(f, g \mid v) \propto p(v \mid f, g) p(f) p(g)
$$

so the probability of each pair depends both on the data - through the likelihood function $p(v \mid f ; g)$ and on the prior probability of the pair itself.

Let us assume that the priors chosen are a good representation of the unknown functions. In this case, any $f$ that does not look like" the unknown function will have a low $p(f)$ and will have a small influence on the posterior estimate. Similarly, any g that does not look like" the unknown function will have a low $p(g)$; in particular, it is unlikely that $g=v / f$ will have high probability for most choices of $f$. Thus, even though such undesirable combinations are not completely discarded, they will have low posterior probability and will only marginally contribute to the posterior mean. It is of course critical that the priors are a good representation of the unknown functions. For Gaussian processes this is determined by the properties of the kernel. One of the main contributions in this paper is the new kernel (14) which is sufficiently flexible so that it can encode the different kinetics (6) while other functions are given low probability.

Also for Gaussian processes the input excitation is very important in order to obtain accurate estimates. The analysis of the effects of the input signal on Bayesian estimates and the choice of optimal input signals fall under the umbrella term of Bayesian input design (Chaloner and Verdinelli, 1995; Vanlier et al., 2012).

Finally, we remark that if data, in terms of $p(v \mid f, g)$, strongly favors a model with low prior probability then such a model will contribute significantly to the estimate. This is in contrast to parametric models for which the model is constrained no matter what the data indicates.

\subsection{Summary of the algorithm}

An outline of our proposed algorithm for the case of $m$ metabolites is as follows:

1. Initialize the parameters $\Theta$ in (17).

2. Use the EM algorithm together with Gibbs sampling to obtain an estimate $\Theta_{\star}$ of $\Theta$.

3. Use Gibbs sampling to obtain the GP estimates $\left\{\hat{h}_{i}\right\}_{i=1}^{m}$ in $(27)$.

4. Minimize the regularized cost in (30) to obtain $\left\{\hat{k}_{i, a c t}, \hat{k}_{i, i n h}, \hat{\gamma}_{i}\right\}_{i=1}^{m}$.

5. Fine tune the parameter values in (31) according to the procedure above.

A Matlab implementation of this algorithm is provided in Github ${ }^{1}$ which can be easily used to reproduce the results of this paper.

\footnotetext{
${ }^{1}$ https://github.com/minglwang/Multilinear_Gaussian_Process
} 


\section{Results}

In this section, we thoroughly study our method through two simulations: (i) numerical experiments are designed to illustrate how the proposed method identifies the modulation effect of each metabolite; (ii) fed-batch simulations using a model from the literature are conducted to validate our method in a more realistic case.

\subsection{Numerical experiments}

In this study, we use two numerical experiments to demonstrate our method. In Experiment 1 , we use the model

$$
v[t]=v_{\max } \frac{c_{1}[t]}{c_{1}[t]+k_{1}} \frac{k_{2}}{c_{2}[t]+k_{2}}
$$

to describe the true reaction kinetics, where $v_{\max }=1, k_{1}=12, k_{2}=56, c_{1}$ and $c_{2}$ have an activation effect $h_{1}$ and an inhibition effect $h_{2}$, respectively. The available information is the data $\left\{y[t], c_{1}[t], c_{2}[t]\right\}_{t=1}^{N}$, where $y[t]$ is the measurement of $v[t]$. Our task is to identify modulation effects of $c_{1}$ and $c_{2}$ from the four candidates shown in Figure 1. We use a nonparameteric GP model with bilinear structure as in (15). The experimental settings are as follows:

- The inputs $c_{1}[t]$ and $c_{2}[t]$ are uniformly sampled from the interval $[1,100]$ and $[1,300]$, respectively. The sample size is set to $N=100$.

- The measurements $y[t]$ are contaminated by white Gaussian noise $e[t]$ with variance $\sigma^{2}=0.1 \operatorname{Var}(v)$, where $\operatorname{Var}(v)$ is the variance of $v[t]$.

- $\mathcal{D}_{1}, \cdots, \mathcal{D}_{n_{r}}$ are $n_{r}$ independent sets of data generated to compare the overall performance. Here $n_{r}=50$.

- The goodness of fit is defined as

$$
\text { Fit }=1-\frac{\|\hat{\boldsymbol{v}}-\boldsymbol{v}\|}{\|\boldsymbol{v}-\overline{\boldsymbol{v}}\|}
$$

where $\hat{\boldsymbol{v}}=[\hat{v}[1], \ldots, \hat{v}[N]]^{T}$ are the estimates calculated by (28), $\boldsymbol{v}=[v[1], \ldots, v[N]]^{T}$ is the vector of true values (33), $\overline{\boldsymbol{v}}$ is the mean value of $\boldsymbol{v}$, Fit $=1$ corresponds to the perfect case where $\hat{\boldsymbol{v}}=\boldsymbol{v}$.

- The selection rate $s_{i}$ for the modulation effect of the metabolite $i$ is defined as the fraction of the $n_{r}$ trials where the correct modulation effect is selected in Step 2. We use $\epsilon=10^{-3}$ as threshold for considering $k_{a c t}$ and $k_{i n h}$ to be zero.

Now, we apply the multilinear GP (bilinear in this case) to the $n_{r}$ data sets $\mathcal{D}_{1}, \cdots, \mathcal{D}_{n_{r}}$. Typical estimates of $h_{1}, h_{2}$ and $v[t]$ using the multilinear GP model are presented in (a), (b) and (c) of Figure 4, respectively. The goodness of fit in (34) is $0.81 \pm 0.052$. This indicates 

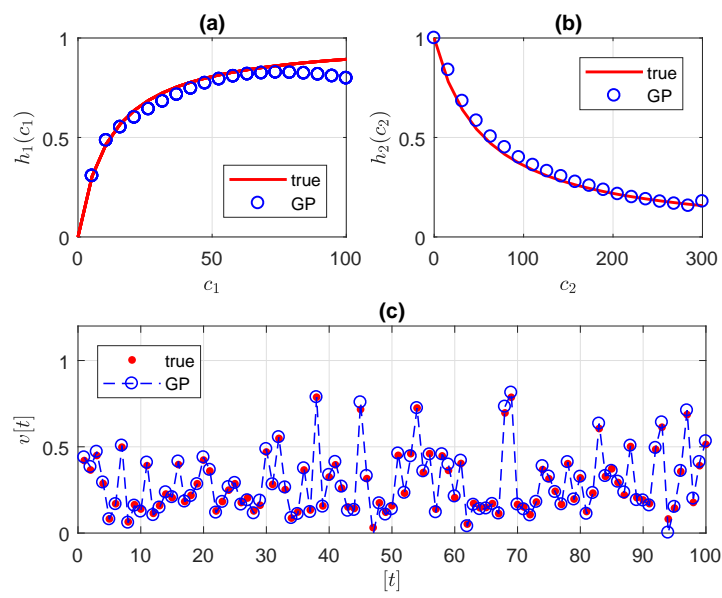

Figure 4: Results for Experiment 1: (a) estimates of $h_{1}$, (b) estimates of $h_{2},(\mathrm{c})$ estimates of $v[t]$, "GP" refers to the estimates from multilinear GP.
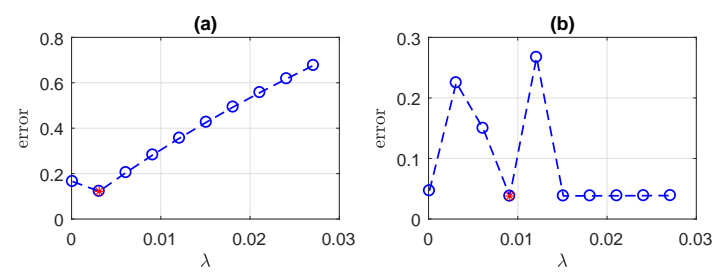

Figure 5: Cross validation: (a) selection of $\lambda$ for $h_{1}$; (b) selection of $\lambda$ for $h_{2}$; red $\star$ refers to the $\lambda$ with the best validation performance.

that our non-parametric GP model well captures the dynamics of the data. Based on the estimates $\hat{h}_{1}, \hat{h}_{2}$ in Figure 4 , we can conjecture, by visual inspection, that the concentration $c_{1}$ has an activation effect and $c_{2}$ has an inhibition effect. This matches the true model.

In the next step, we need to fit a Monod-type model to the GP estimates $\hat{h}_{1}\left(c_{1}\right)$ and $\hat{h}_{2}\left(c_{2}\right)$. For comparison, we use the standard quadratic cost in (29) to fit the GP estimates for the 50 data sets. The initial values for $\left\{\gamma_{i}, k_{i, a c t}, k_{i, i n h}\right\}$ are identically $\{1,1,1\}$. The mean values of the identified parameters are presented in Table 1 and are far from the true ones. Despite this, the cost (29) of the standard quadratic approach is often satisfactory. This means the standard approach can be trapped in local optima. This is partly due to the over-parameterized nature of $h_{d c}$ as argued in Section 2.

As an alternative, starting with the same initial values used in the above standard approach, we applied the proposed $\ell_{1}$-regularization scheme using the multilinear GP models as "data". The Matlab function "fmincon" is used to optimize the cost function (30). The penalty factor $\lambda$ is selected by a 5 -fold cross validation $(\mathrm{C}-\mathrm{V})$. The details of $\mathrm{K}$-fold cross validation can be found in Appendix C. The cross validation process for choosing $\lambda$ is presented in Figure 5. The mean values of identified parameters (for the 50 data sets) are 
listed in Table 1 which are close to the true values. We also check the selection rate of the regularized approach. The results are presented in Table 2 , where $\mathrm{C}-\mathrm{V}$ denotes the results obtained using the $\lambda$ selected by cross validation. The selection rates of $h_{1}$ and $h_{2}$ are relatively low. This is because the values of the parameters $k_{1, i n h}, k_{2, a c t}$, which ought to be shrunk to zero, remain larger than $\epsilon$. This indicates that the K-fold $\mathrm{C}-\mathrm{V}$ has not penalized the trivial parameters enough, giving priority to model fitting.

Table 1: Identified parameters in Experiment 1.

\begin{tabular}{lccccr}
\hline & $v_{\max }$ & $k_{1, a c t}=k_{1}$ & $k_{1, \text { inh }}$ & $k_{2, a c t}$ & $k_{2, \text { inh }}=1 / k_{2}$ \\
\hline True & 1 & 12 & 0 & 0 & 0.0179 \\
$\ell_{1}$-RIS (C-V) & 0.8283 & 12.8423 & 0.0007 & 8.4526 & 0.1029 \\
$\ell_{1}$-RIS (E-C) & 0.5663 & 5.8566 & 0 & 0 & 0.0174 \\
Fine-tuned & 0.9904 & 11.1255 & 0 & 0 & 0.0180 \\
Direct-Opt & $1.98 \times 10^{4}$ & 11.05 & $2.29 \times 10^{4}$ & 49.8557 & 388.7420 \\
Direct-Opt (with C-V RIS) & 3.4566 & 11.2581 & 0.0001 & 2.7491 & 0.0718 \\
Direct-Opt (with E-C RIS) & 0.7745 & 0.0695 & 0 & 0.0013 & 0.0177 \\
\hline
\end{tabular}

Table 2: Selection rates of $\ell_{1}$-RIS for the 2 experiments.

\begin{tabular}{lccccr}
\hline Experiment 1 & $s_{1}$ & $s_{2}$ & Experiment 2 & $s_{1}$ & $s_{2}$ \\
\hline C-V & $80 \%$ & $84 \%$ & C-V & $96 \%$ & $80 \%$ \\
E-C & $96 \%$ & $88 \%$ & E-C & $100 \%$ & $84 \%$ \\
\hline
\end{tabular}

A possible solution is to change $\epsilon$ to a larger value. However, it is hard to find such a value in a systematic way. A better option is to increase the penalty factor $\lambda$ at the cost of slightly sacrificing the goodness of fit. In this paper, an empirical choice (E-C) for a larger $\lambda$ is $1 \times 10^{-3} \bar{c}_{i}$, where $\bar{c}_{i}$ is the mean value of $c_{i}$. One can also use the one standard error rule for better choices of $\lambda$ (Friedman et al., 2001). Using this empirical $\lambda$, the identified parameters are presented in Table 1 which have worse match with the true values when compared with the values from the $\mathrm{C}-\mathrm{V}$ approach. However, the selection rate is significantly improved as demonstrated in Table 2. Thus, this empirical choice of $\lambda$ is adopted. Then we combine the identified models into a complete model as in (31). Finally, we fine tune the model parameters using the NLLS implemented by the Matlab function "lsqnonlin". As shown in Table 1, the fine-tuned parameter values are very close to their true values.

In addition, we compare our algorithm with a direct optimization approach (DirectOpt). The Direct-Opt approach uses the Monod-type function $h_{d c}$ as a general model for all metabolites and combine them to one complete model as (31), then optimize all parameters at once. The initial values are set to be the same as in the proposed approach. The identified parameters of this approach are listed in Table 1 for comparison. We can see that they are far from the true values. Regularization can also be used in the Direct-Opt approach and the second last row in Table 1 lists the sample means obtained using $\mathrm{C}-\mathrm{V}$. The mean values of results from 50 data sets are list in Table 1. This approach has difficulty in shrinking the 

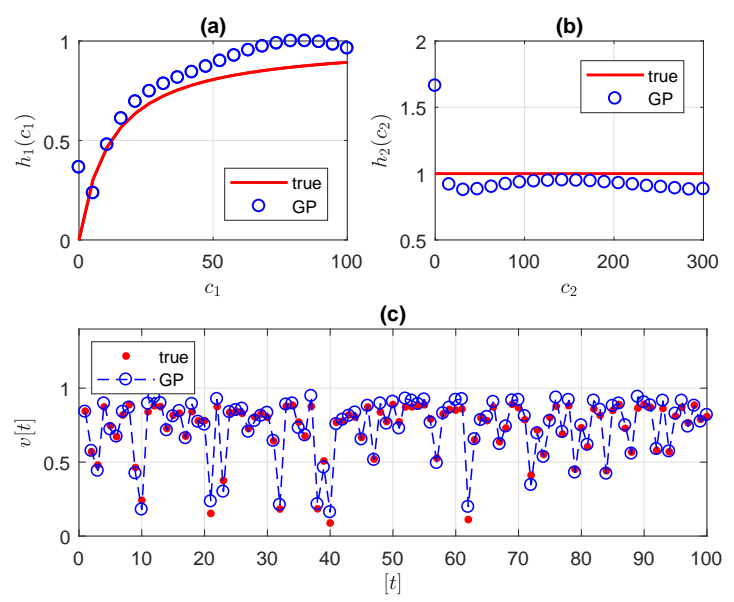

Figure 6: Results for Experiment 2: (a) estimates for $h_{1}\left(c_{1}\right)$, "GP" refers to the estimate from multilinear GP. (b) estimates for $h_{2}\left(c_{2}\right) ;(\mathrm{c})$ the estimates for $v[t]$.

trivial parameters to 0 . In order to see if we can improve the fit by increasing the penalty $\lambda$ we have also tried the empirical choice $\lambda\left(\min \left(1 \times 10^{-3} \bar{c}_{i}\right)\right)$ discussed above. The results are shown in the last row of Table 1 and indicate that this method does not provide a penalty large enough to shrink the trivial parameter $k_{a c t, 1}$ to 0 yet penalizes the key factor $k_{a c t, 1}$ too much and make it very close to 0 . Thus, for the Direct-Opt approach, we can not penalize the right parameters by simply tuning the penalty factor $\lambda$. However, the proposed penalty term is quite beneficial in avoiding the local optima of the Direct-Opt approach.

We also tested the performance of the Gaussian noise model assumption when the underlying distribution is otherwise. For example, given the same settings, the observations of reaction rate $v$ are contaminated by the independently and uniformly distributed noise $\mathcal{U}\left[-0.1 v_{\max }, 0.1 v_{\max }\right]$, the performance of our method is given in Figure 7 . The figure shows that the GP are robust to capture the modulation types of each metabolite.

A second experiment is designed to show that the proposed algorithm neglects trivial ("neutral") effects in noisy data. In this experiment, we set the true model as

$$
v[t]=v_{\max } \frac{c_{1}[t]}{c_{1}[t]+k_{1}},
$$

i.e. $c_{2}$ has no effect, and use the same simulation settings as Experiment 1. We check the performance of our algorithm step by step as in Experiment 1. For Step 1, the GP estimates are presented in Figure 6. The estimates properly indicate the modulation effects of the metabolites. The goodness of fit is calculated via (34), Fit $=0.81 \pm 0.002$. Based on the estimates $\hat{h}_{1}, \hat{h}_{2}$, we apply the Step $2\left(\ell_{1}\right.$-RIS) to the data sets. The identified parameters are shown in Table 3. The corresponding selection rates are presented in Table 2 . The results demonstrate the ability of $\ell_{1}$-RIS to neglect the trivial components in the model. For Step 3 , the fine-tuned parameters are presented in Table 3 which match the true values. The parameters identified by the direct optimization approach are also listed in Table 3 which, 

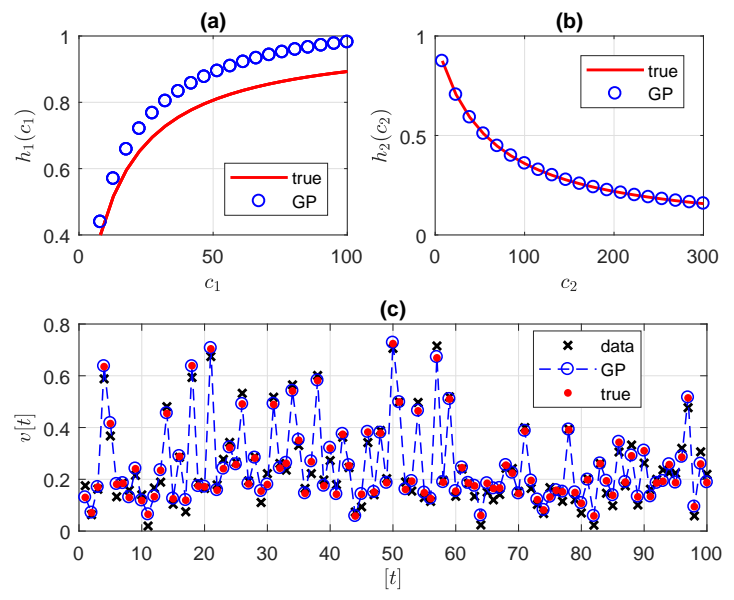

Figure 7: GP estimates under uniformly distributed noise with Gaussian noise model assumption

similar to Experiment 1, have a poor match with the true values.

Table 3: Identified parameters in Experiment 2.

\begin{tabular}{lccccc}
\hline & $v_{\max }$ & $k_{1, a c t}=k_{1}$ & $k_{1, \text { inh }}$ & $k_{2, a c t}$ & $k_{2, \text { inh }}$ \\
\hline True & 1 & 12 & 0 & 0 & 0 \\
$\ell_{1}$-RIS (C-V) & 1.5124 & 12.0414 & 0.0001 & 0.0198 & 0 \\
$\ell_{1}$-RIS (E-C) & 1.3403 & 8.6285 & 0 & 0 & 0 \\
Fine-tuned & 1.0026 & 12.1013 & 0 & 0 & 0 \\
Direct-Opt & $7.99 \times 10^{4}$ & 1177.2107 & 0.0598 & 6606.4712 & 1.6712 \\
Direct-Opt (with CV RIS) & 0.9695 & 9.7498 & 0 & 0.0526 & 0 \\
Direct-Opt (with EC RIS) & 0.8743 & 4.3184 & 0 & 0.1327 & 0.0000 \\
\hline
\end{tabular}

\subsection{Fed-batch experiments}

In this section, we study the performance of our method in simulated fed-batch experiments. The studied bioprocess consists of a culture of bacteria (B. subtilis). It consumes substrates (carbon and nitrogen) to produce an enzyme. Following the work in (Grosfils et al., 2007), the reaction scheme is given as

$$
\begin{aligned}
& 1 \mathrm{C} \stackrel{v_{1}}{\rightarrow} 0.2 \mathrm{X}_{\text {non-lysed }}, \\
& 1 \mathrm{~N} \stackrel{v_{2}}{\rightarrow} 15 \mathrm{X}_{\text {non-lysed }}+120 \mathrm{E}, \\
& 1 \mathrm{X}_{\text {non-lysed }} \stackrel{v_{3}}{\rightarrow} \mathrm{X}_{\text {lysed }},
\end{aligned}
$$

where $\mathrm{X}_{\text {non-lysed }}, \mathrm{X}_{\text {lysed }}, \mathrm{E}, \mathrm{C}, \mathrm{N}$ are the non-lysed cells, lysed cells, enzymes, carbon and nitrogen concentrations; $v_{1}, v_{2}, v_{3}$ are the reaction rates. According to (Bogaerts et al., 
Table 4: Experimental settings for Fed-batch experiments: initial concentrations and substrate concentrations of the feed medium $(\mathrm{g} / \mathrm{l})$.

\begin{tabular}{lllllll}
\hline & $\mathrm{C}_{\text {init }}$ & $\mathrm{N}_{\text {init }}$ & $\mathrm{C}^{\text {in }}$ & $\mathrm{N}^{\text {in }}$ & $F\left(\mathrm{~h}^{-1}\right)$ & $D\left(\mathrm{~h}^{-1} \mathrm{l}^{-1}\right)$ \\
\hline 1 & 11.6 & 0.4 & 166.9 & 10.8 & & \\
2 & 11.3 & 0.5 & 100 & 6.7 & & \\
3 & 10.1 & 0.5 & 33.8 & 2.7 & & \\
4 & 11.4 & 0.6 & 1 & 6.7 & 0.091 & 0.02275 \\
5 & 50.1 & 0.5 & 33.8 & 10.8 & & \\
6 & 1 & 0.5 & 33.8 & 10.8 & & \\
7 & 11.7 & 0.6 & 67.5 & 6.7 & & \\
\hline
\end{tabular}

1999), the kinetics of the reaction rates can be modeled as

$$
\begin{aligned}
v_{1} & =v_{1, \max } \frac{c_{\mathrm{X}}}{k_{1, \mathrm{X}}+c_{\mathrm{X}}} \frac{c_{\mathrm{C}}}{k_{1, \mathrm{C}}+c_{\mathrm{C}}} \frac{k_{1, \mathrm{C}}^{\prime}}{c_{\mathrm{C}}+k_{1, \mathrm{C}}^{\prime}} \frac{c_{\mathrm{N}}}{c_{\mathrm{N}}+k_{1, \mathrm{~N}}}, \\
v_{2} & =v_{2, \max } \frac{c_{\mathrm{X}}}{k_{2, \mathrm{X}}+c_{\mathrm{X}}} \frac{c_{\mathrm{C}}}{k_{2, \mathrm{C}}+c_{\mathrm{C}}} \frac{k_{2, \mathrm{C}}^{\prime}}{c_{\mathrm{C}}+k_{2, \mathrm{C}}^{\prime}} \frac{c_{\mathrm{N}}}{c_{\mathrm{N}}+k_{2, \mathrm{~N}}} \frac{k_{2, \mathrm{E}}^{\prime}}{c_{\mathrm{E}}+k_{2, \mathrm{E}}^{\prime}},
\end{aligned}
$$

where $c_{\mathrm{X}}, c_{\mathrm{C}}, c_{\mathrm{N}}, c_{\mathrm{E}}$ are the concentrations of $\mathrm{X}, \mathrm{C}, \mathrm{N}, \mathrm{E}$, respectively, $\mathrm{X}$ is short for $\mathrm{X}_{\text {non-lysed }}$, and $k^{\prime}$ denotes parameters for inhibition effects. The kinetics of reaction (38) is proportional to the concentration of $\mathrm{X}_{\text {non-lysed }}$,

$$
v_{3}=0.051 \mathrm{X}_{\text {non-lysed }}
$$

where 0.051 is the so called lysis rate. In this study, we are only interested in the kinetics of $v_{1}$ and $v_{2}$. Though their kinetic structures can be deduced from the reaction scheme using the biological knowledge, we assume they are unknown in this study in order to test the performance of our method. So our task is to determine the unknown kinetics of $v_{1}$ and $v_{2}$.

Using the law of mass balance, the kinetic model of the fed-batch process can be formulated as

$$
\frac{d \boldsymbol{c}[t]}{d t}=\boldsymbol{S} \boldsymbol{v}[t]-D[t] \boldsymbol{c}[t]+D[t] \boldsymbol{c}^{\mathrm{in}}
$$

where $\boldsymbol{c}=\left[c_{\mathrm{X}}, c_{\mathrm{C}}, c_{\mathrm{N}}, c_{\mathrm{E}}\right]^{T}, \boldsymbol{v}=\left[v_{1}, v_{2}\right]^{T}, \boldsymbol{S}$ is the stoichiometric matrix which can be readily obtained from $(36)-(38), c^{\text {in }}$ is the substrate concentration of the feed medium, $D[t]=$ $F[t] / V$ is the dilution rate, $F[t]$ is the feeding rate, $V$ is the volume of culture media.

In this simulation study, we set up 7 fed-batch experiments. The initial conditions and substrate concentrations of feed medium for each experiments are given in Table 4. The parameter values are given in Table 5 . The total culture time is $39 \mathrm{~h}$ and we take samples every $4 \mathrm{~h}$. The data generated by the first six experiments are used for training and the remaining data used for testing. These settings are similar to (Grosfils et al., 2007). 
Considering the 4 metabolites in the reactions, we set up our multilinear model for each reaction rate as

$$
y[t]=\prod_{j=1}^{4} h_{j}\left(c_{j}[t], k_{j}\right)+e[t],
$$

where the subscript $j$ is associated to one of the elements of $[\mathrm{X}, \mathrm{C}, \mathrm{N}, \mathrm{E}], e_{i}[t] \sim \mathcal{N}\left(0,0.1 \operatorname{Var}\left(v_{i}\right)\right)$, $i=1,2$.
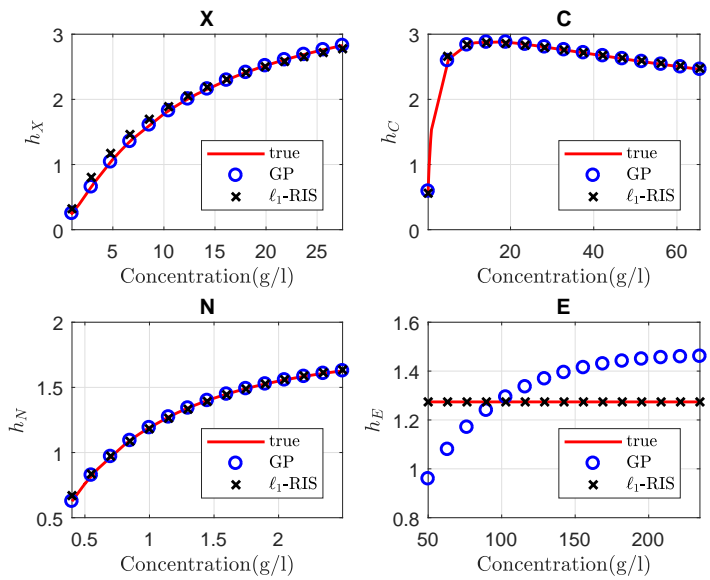

Figure 8: Multilinear GP estimates and $\ell_{1}$-RIS results for the kinetics in (39).
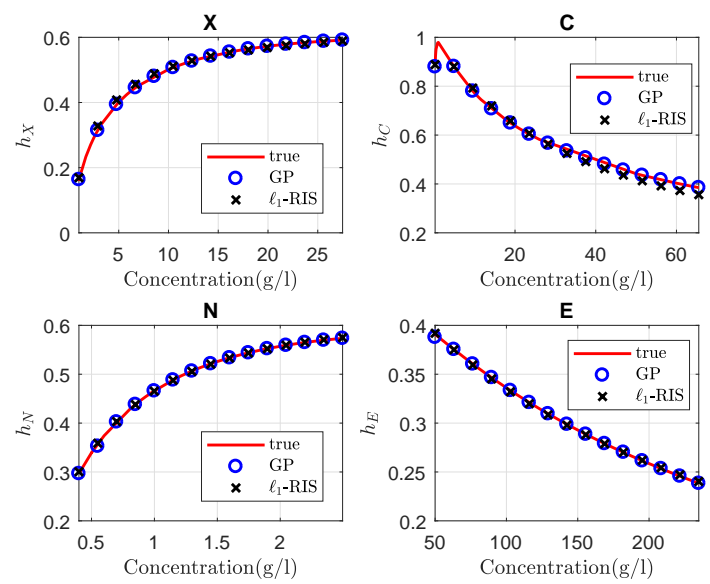

Figure 9: Multilinear GP estimates and $\ell_{1}$-RIS results for the kinetics in (40).

The results of multilinear GP estimation and the $\ell_{1}$-RIS are presented in Figure 8 and Figure 9. By visual inspection, we see that the estimates from the multilinear GP correctly 

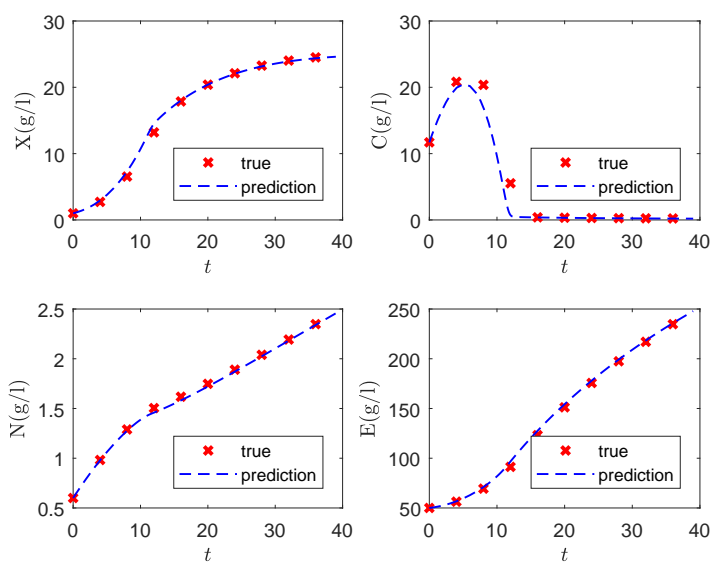

Figure 10: The prediction result of the fed-batch experiment 7 with the fine-tuned parameters in Table 5 .

Table 5: Identified parameters: key parameters.

\begin{tabular}{lllllll}
\hline reaction 1 & $v_{\max }$ & $k_{\mathrm{X}}$ & $k_{\mathrm{C}}$ & $k_{\mathrm{N}}$ & & $k_{\mathrm{C}}^{\prime}$ \\
True & 181 & 9.9 & 1.02 & 12.1 & & 82.7 \\
$\ell_{1}$-RIS & 54 & 11.98 & 0.86 & 1.54 & & 156.25 \\
Fine-tuned & 157.70 & 9.21 & 0.86 & 10.64 & & 85.49 \\
\hline reaction 2 & $v_{\max }$ & $k_{\mathrm{X}}$ & $k_{\mathrm{C}}$ & $k_{\mathrm{N}}$ & $k_{\mathrm{E}}^{\prime}$ & $k_{\mathrm{C}}^{\prime}$ \\
True & 0.5 & 4.0 & 0.01 & 1.47 & 69.6 & 27.1 \\
$\ell_{1}$-RIS & 0.36 & 2.63 & 0.01 & 1.25 & 96.78 & 27.70 \\
Fine-tuned & 0.45 & 4.38 & 0.05 & 1.20 & 70.8 & 28.60 \\
\hline
\end{tabular}

reveal the modulation effect of each metabolite except the estimate of $\mathrm{E}$ in the first reaction (36). Subsequently, the $\ell_{1}$-RIS is applied to shrink the trivial effects so that we can maintain a minimum number of components in our final model. As demonstrated by Figure 8 and Figure 9, the selected modulation functions for each metabolite match with the true kinetic models in (39) and (40), e.g. in Figure 8, the results of $\ell_{1}$-RIS (the cross in black) indicate that $\mathrm{X}$ and $\mathrm{N}$ have an activation effect, $\mathrm{C}$ has a double-component effect and $\mathrm{E}$ has "neutral" effect.

The parameters identified by $\ell_{1}$-RIS are given in Table 5 and Table 6 . All the trivial parameters identified by $\ell_{1}$-RIS are very close to zero except $1 / k_{N}^{\prime}>\epsilon=10^{-3}$. However, this does not affect our final result too much. The fine-tuned parameters are given in Table 5 which match with the reference values in the same table.

In addition, we test the estimated model using the fine-tuned parameters on the testing data. The results are presented in Figure 10 where it can be verified that the model fit is very good also on this data set which has not been used for estimation. 
Table 6: Identified parameters: trivial parameters.

\begin{tabular}{lllll}
\hline reaction 1 & $1 / k_{\mathrm{X}}^{\prime}$ & $1 / k_{\mathrm{N}}^{\prime}$ & $k_{\mathrm{E}}$ & $1 / k_{\mathrm{E}}^{\prime}$ \\
$\ell_{1}$-RIS & $1.29 \times 10^{-7}$ & 0.012 & $4.03 \times 10^{-6}$ & $1.77 \times 10^{-9}$ \\
\hline reaction 2 & $1 / k_{\mathrm{X}}^{\prime}$ & $1 / k_{\mathrm{N}}^{\prime}$ & $k_{\mathrm{E}}$ & \\
$\ell_{1}$-RIS & $4.47 \times 10^{-7}$ & $3.78 \times 10^{-7}$ & $4.17 \times 10^{-6}$ & \\
\hline
\end{tabular}

\section{Conclusion}

In this paper, we proposed a three step procedure for estimating kinetics for macroscopic reactions. First, one Gaussian process was used to model each metabolite contribution to the reaction rate. Instrumental to this end was the new kernel (14) which is tailored to represent common kinetics. Subsequently, in Step 2, Monod models were estimated from these non-parametric models. Here, $\ell_{1}$-regularization was used to incorporate automatic selection of which type of contribution (activation, inhibition, double or neutral) that each metabolite has. In this step cross-validation plays an important role. Finally, the parameters in the Monod model are fine tuned by simultaneously tuning all the parameters based on the model for the entire reaction. It is important to notice that the last step is greatly facilitated by the previous steps for two reasons: 1) the type of effect each metabolite has (activation, etc) has been determined, and 2) initial values for the non-zero parameters have been obtained. This should be compared with trying to directly optimize a Monod-type model in which case (without prior knowledge) the model has to allow for all types of effects for all metabolites, leading to a very complex model, and finding reasonable initial values for the parameters can be very difficult, in turn leading to difficulties when tuning (optimizing) the parameters.

Two numerical experiments have been conducted to demonstrate that the non-parametric multilinear GP is able to fully capture the kinetics of the data. In addition, Experiment 1 showed that the multilinear GP can estimate the activation and inhibition effects correctly in a bilinear structure, while Experiment 2 validated that the proposed $\ell_{1}$-RIS ignores neutral components in the model. Fed-batch experiments showed the ability of the algorithm to deal with a multilinear Monod model with 4 components. The selected model and parameters gave satisfactory prediction results. In conclusion, the proposed algorithm is a novel contribution to deal with the model selection and parameter estimation problem in identifying the nonlinear kinetics of biological systems.

\section{Funding}

This work was supported by the VINNOVA Competence Centre AdBIOPRO, contract [2016-05181], and by the Swedish Research Council through the research environment NewLEADS - New Directions in Learning Dynamical Systems, contract [2016-06079].

\section{Appendix A. Maximum likelihood estimation}

In this appendix we study maximum likelihood estimation in the model (7) given observations $\boldsymbol{y}=\left[\begin{array}{lll}y_{1} & \ldots & y_{N}\end{array}\right]^{T}$ assuming that the measurement errors $\left\{e_{i}\right\}_{i=1}^{N}$ are i.i.d. $\mathcal{N}\left(0, \sigma_{e}^{2}\right)$ and $f$ is a GP with mean 0 and kernel $K_{\theta}$. 
From the definition of a GP we have that

$$
\boldsymbol{f}:=\left[\begin{array}{c}
f\left(\boldsymbol{x}_{1}\right) \\
\vdots \\
f\left(\boldsymbol{x}_{N}\right)
\end{array}\right] \sim \mathcal{N}\left(0, \boldsymbol{K}_{\theta}\right)
$$

Furthermore, $\boldsymbol{e}:=\left[\begin{array}{lll}e_{1} & \ldots & e_{N}\end{array}\right]^{T} \sim \mathcal{N}\left(0, \sigma_{e}^{2} I\right)$ and hence (7) implies that $\boldsymbol{y}$ is the sum of two normal vectors and therefore normal distributed as well. Using that $\boldsymbol{f}$ and $\boldsymbol{e}$ are independent gives

$$
\boldsymbol{y}=\boldsymbol{f}+\boldsymbol{e} \sim \mathcal{N}\left(\mathbf{0}, \boldsymbol{K}_{\theta}+\sigma_{e}^{2} \boldsymbol{I}_{N}\right)
$$

The Empirical Bayes estimate $\theta_{\star}$ of $\theta$ is defined as the maximizer of the corresponding likelihood $\mathcal{N}\left(\boldsymbol{y} ; \mathbf{0}, \boldsymbol{K}_{\theta}+\sigma_{e}^{2} \boldsymbol{I}_{N}\right)$.

Assuming $\theta=\theta_{\star}$, the posterior distribution of $\boldsymbol{f}$ can be computed using the Bayes' theorem,

$$
\mathrm{p}(\boldsymbol{f} \mid \boldsymbol{y})=\frac{\mathrm{p}(\boldsymbol{y} \mid \boldsymbol{f}) \mathrm{p}\left(\boldsymbol{f} ; \theta_{\star}\right)}{\mathrm{p}\left(\boldsymbol{y} ; \theta_{\star}\right)}
$$

resulting in $\mathrm{p}\left(\boldsymbol{f} \mid \boldsymbol{y} ; \theta_{\star}\right)=\mathcal{N}\left(\boldsymbol{f} ; u_{\star}, \Sigma_{\star}\right)$ where

$$
\mu_{\star}=\boldsymbol{K}_{\theta_{\star}}\left(\boldsymbol{K}_{\theta_{\star}}+\sigma_{e}^{2} \boldsymbol{I}_{N}\right)^{-1} \boldsymbol{y}, \quad \Sigma_{\star}=\boldsymbol{K}_{\theta_{\star}}-\boldsymbol{K}_{\theta_{\star}}\left(\boldsymbol{K}_{\theta_{\star}}+\sigma_{e}^{2} \boldsymbol{I}_{N}\right)^{-1} \boldsymbol{K}_{\theta_{\star}}
$$

The posterior for $f$ evaluated at other values of $\boldsymbol{x}$ can also be computed but we refer to (Rasmussen, 2004) for more details on Gaussian processes.

\section{Appendix B. Implementation of Gibbs sampling and the EM algorithm}

The details of Gibbs sampling at the $n_{\text {th }}$ step of the EM-algorithm are given as follows:

1. Initialize $\boldsymbol{f}=\boldsymbol{f}_{0}, \boldsymbol{g}=\boldsymbol{g}_{0}, l=l_{b}$;

2. Sample $\boldsymbol{g}_{l} \sim \mathrm{p}\left(\boldsymbol{g} \mid \boldsymbol{f}_{l-1}, \boldsymbol{y} ; \beta_{n}\right)$ using (24);

3. Sample $\boldsymbol{f}_{l} \sim \mathrm{p}\left(\boldsymbol{f} \mid \boldsymbol{g}_{l}, \boldsymbol{y} ; \alpha_{n}\right)$ using $(25), l=l+1$;

4. Iterate Steps 2 and 3.

Note that $l_{b}<0$ is designed for the burn-in period of Gibbs sampling. We take $\boldsymbol{g}_{l}, \boldsymbol{f}_{l}, l=$ $1, \cdots, M$ as our samples.

The EM algorithm using Gibbs sampling is implemented as follows:

While $n<n_{\max }$

1. Calculate the Gram matrices $\boldsymbol{K}_{\alpha}$ and $\boldsymbol{K}_{\beta}$ using parameter $\Theta_{n}$

2. Sample $\left\{\boldsymbol{g}_{l}, \boldsymbol{f}_{l}\right\}_{l=1}^{M}$ using the Gibbs sampling above

3. Compute $\hat{Q}_{n, M}(\Theta)$ in (23) and maximize this function to get $\Theta_{n+1}$

4. If $\left\|\Theta_{n+1}-\Theta_{n}\right\|^{2}<\Delta$, return $\Theta^{\star}=\Theta_{n+1}$ 


\section{Appendix C. K-fold cross validation}

The K-fold cross validation is conducted as follows (Friedman et al., 2001):

1. Divide the set $\{1, \cdots, t, \cdots, N\}$ into $\mathrm{K}$ subsets of equal size, $D_{1}, \cdots, D_{K}$.

For $n=1, \cdots, \mathrm{K}$ :

(a) Denote the parameters minimizing the regularized cost

$$
\begin{aligned}
& \sum_{t \notin D_{n}}\left[\hat{h}_{i}[t]-\gamma h_{d c}\left(c_{i}[t] ; k_{a c t}, k_{i n h}\right)\right]^{2}+\lambda\left(k_{a c t}+k_{i n h}\right) \\
& \text { s.t. } \quad k_{a c t} \geq 0, k_{i n h} \geq 0
\end{aligned}
$$

by $\hat{k}_{i, a c t}(\lambda), \hat{k}_{i, i n h}(\lambda)$ and $\hat{\gamma}_{i}(\lambda)$.

(b) Calculate the validation error,

$$
e_{i, n}(\lambda)=\sum_{t \in D_{n}}\left(\hat{h}_{i}[t]-\hat{\gamma}_{i}(\lambda) h_{d c}\left(c_{i}[t] ; \hat{k}_{i, a c t}(\lambda), \hat{k}_{i, i n h}(\lambda)\right)\right)
$$

2. Compute the average error over all $\mathrm{K}$ folds,

$$
\mathrm{CV}_{i}(\lambda)=\frac{1}{N} \sum_{n=1}^{\mathrm{K}} e_{i, n}(\lambda)
$$

3. Return $\lambda_{i}:=\arg \min _{\lambda \geq 0} \mathrm{CV}_{i}(\lambda)$ and $\hat{k}_{i, a c t}\left(\lambda_{i}\right), \hat{k}_{i, i n h}\left(\lambda_{i}\right)$ and $\hat{\gamma}_{i}\left(\lambda_{i}\right)$ as parameter estimates for $i=1, \ldots, m$.

\section{References}

Aitchison, J., 1964. Confidence-region tests. Journal of the Royal Statistical Society: Series B (Methodological) $26(3), 462-476$.

Akaike, H., 1998. Information theory and an extension of the maximum likelihood principle. In: Selected papers of hirotugu akaike. Springer, pp. 199-213.

Andrews, J. F., 1968. A mathematical model for the continuous culture of microorganisms utilizing inhibitory substrates. Biotechnology and Bioengineering 10 (6), 707-723.

Batt, B. C., Kompala, D. S., 1989. A structured kinetic modeling framework for the dynamics of hybridoma growth and monoclonal antibody production in continuous suspension cultures. Biotechnology and bioengineering 34 (4), 515-531.

Bogaerts, P., Castillo, J., Hanus, R., 1999. A general mathematical modelling technique for bioprocesses in engineering applications. Systems analysis modelling simulation 35 (2), 87-113.

Bradford, E., Schweidtmann, A. M., Zhang, D., Jing, K., del Rio-Chanona, E. A., 2018. Dynamic modeling and optimization of sustainable algal production with uncertainty using multivariate gaussian processes. Computers \& Chemical Engineering 118, 143-158.

Brunton, S. L., Proctor, J. L., Kutz, J. N., 2016. Discovering governing equations from data by sparse identification of nonlinear dynamical systems. Proceedings of the National Academy of Sciences 113 (15), 3932-3937. 
Carroll, R. J., Ruppert, D., Crainiceanu, C. M., Stefanski, L. A., 2006. Measurement error in nonlinear models: a modern perspective. Chapman and Hall/CRC.

Casella, G., 1985. An introduction to empirical Bayes data analysis. The American Statistician 39 (2), 83-87.

Casella, G., George, E. I., 1992. Explaining the Gibbs sampler. The American Statistician 46 (3), 167-174.

Chaloner, K., Verdinelli, I., 1995. Bayesian experimental design: A review. Statistical Science, 273-304.

Chen, L., Bernard, O., Bastin, G., Angelov, P., 2000. Hybrid modelling of biotechnological processes using neural networks. Control Engineering Practice 8 (7), 821-827.

Del Rio-Chanona, E. A., Cong, X., Bradford, E., Zhang, D., Jing, K., 2019. Review of advanced physical and data-driven models for dynamic bioprocess simulation: Case study of algae-bacteria consortium wastewater treatment. Biotechnology and bioengineering 116 (2), 342-353.

Dempster, A. P., Laird, N. M., Rubin, D. B., 1977. Maximum likelihood from incomplete data via the EM algorithm. Journal of the royal statistical society. Series B (methodological), 1-38.

Dimitrov, S. D., Kamenski, D. I., 1991. A parameter estimation method for rational functions. Computers \& chemical engineering $15(9), 657-662$.

Ding, J., Tarokh, V., Yang, Y., 2018. Model Selection Techniques: An Overview. IEEE Signal Processing Magazine 35 (6), 16-34.

Donoho, D. L., 2006. For most large underdetermined systems of linear equations the minimal L1-norm solution is also the sparsest solution. Communications on Pure and Applied Mathematics: A Journal Issued by the Courant Institute of Mathematical Sciences 59 (6), 797-829.

Fiedler, B., Schuppert, A., 2008. Local identification of scalar hybrid models with tree structure. IMA Journal of Applied Mathematics 73 (3), 449-476.

Friedman, J., Hastie, T., Tibshirani, R., 2001. The elements of statistical learning. Vol. 1. Springer series in statistics New York, NY, USA:.

Grosfils, A., Wouwer, A. V., Bogaerts, P., 2007. On a general model structure for macroscopic biological reaction rates. Journal of biotechnology 130 (3), 253-264.

Grosfils, A., Wouwer, A. V., Ph, B., 2005. Hybrid neural network models of bioprocesses: a comparative study. In: Proceedings of the 16th IFAC World Congress, Prague.

Haag, J. E., Wouwer, A. V., Remy, M., 2005. A general model of reaction kinetics in biological systems. Bioprocess and biosystems engineering 27 (5), 303-309.

Hagrot, E., Oddsdóttir, H. Æ., Hosta, J. G., Jacobsen, E. W., Chotteau, V., 2017. Poly-pathway model, a novel approach to simulate multiple metabolic states by reaction network-based model-Application to amino acid depletion in CHO cell culture. Journal of biotechnology 259, 235-247.

Hagrot, E., Oddsdóttir, H. Æ., Mäkinen, M., Forsgren, A., Chotteau, V., 2019. Novel column generationbased optimization approach for poly-pathway kinetic model applied to CHO cell culture. Metabolic engineering communications 8, e00083.

Hahs-Vaughn, D. L., Lomax, R. G., 2013. Statistical concepts: A second course. Routledge.

Heiser, R. F., Parrish, W. R., 1989. Representing physical data with rational functions. Industrial \& engineering chemistry research 28 (4), 484-489.

Kohavi, R., Others, 1995. A study of cross-validation and bootstrap for accuracy estimation and model selection. In: Ijcai. Vol. 14. Montreal, Canada, pp. 1137-1145.

Lennart, L., 1999. System identification: theory for the user. PTR Prentice Hall, Upper Saddle River, NJ, $1-14$.

Leskovac, V., 2003. Comprehensive enzyme kinetics. Springer Science \& Business Media.

Lillacci, G., Khammash, M., 2010. Parameter estimation and model selection in computational biology. PLoS computational biology 6 (3), e1000696.

Mailier, J., Wouwer, A. V., 2012. Identification of nested biological kinetic models using likelihood ratio tests. Chemical engineering science 84, 727-734.

Mangan, N. M., Brunton, S. L., Proctor, J. L., Kutz, J. N., 2016. Inferring biological networks by sparse identification of nonlinear dynamics. IEEE Transactions on Molecular, Biological and Multi-Scale Communications 2 (1), 52-63.

Marquardt, D. W., 1963. An algorithm for least-squares estimation of nonlinear parameters. Journal of the 
society for Industrial and Applied Mathematics 11 (2), 431-441.

McLachlan, G., Krishnan, T., 2007. The EM algorithm and extensions. Vol. 382. John Wiley \& Sons.

Menten, L., Michaelis, M. I., 1913. Die kinetik der invertinwirkung. Biochem Z 49, 333-369.

Oliveira, R., 2004. Combining first principles modelling and artificial neural networks: a general framework. Computers \& Chemical Engineering 28 (5), 755-766.

Olofsson, S., Hebing, L., Niedenführ, S., Deisenroth, M. P., Misener, R., 2019. Gpdoemd: A python package for design of experiments for model discrimination. Computers \& Chemical Engineering 125, 54-70.

Pagan, A. R., Hall, A. D., 1983. Diagnostic tests as residual analysis. Econometric Reviews 2 (2), $159-218$.

Park, M. Y., Hastie, T., 2007. L1-regularization path algorithm for generalized linear models. Journal of the Royal Statistical Society: Series B (Statistical Methodology) 69 (4), 659-677.

Rasmussen, C. E., 2004. Gaussian processes in machine learning. In: Advanced lectures on machine learning. Springer, pp. 63-71.

Richelle, A., Bogaerts, P., 2015. Systematic methodology for bioprocess model identification based on generalized kinetic functions. Biochemical engineering journal 100, 41-49.

Savageau, M. A., 1969a. Biochemical systems analysis: I. some mathematical properties of the rate law for the component enzymatic reactions. Journal of theoretical biology 25 (3), 365-369.

Savageau, M. A., 1969b. Biochemical systems analysis: Ii. the steady-state solutions for an n-pool system using a power-law approximation. Journal of theoretical biology 25 (3), 370-379.

Schmidt, M., 2005. Least squares optimization with L1-norm regularization. CS542B Project Report, 14-18.

Schwarz, G., Others, 1978. Estimating the dimension of a model. The annals of statistics 6 (2), 461-464.

Strutz, T., 2010. Data fitting and uncertainty: A practical introduction to weighted least squares and beyond. Vieweg and Teubner.

Tibshirani, R., 1996. Regression shrinkage and selection via the lasso. Journal of the Royal Statistical Society: Series B (Methodological) 58 (1), 267-288.

Toulias, T. L., Kitsos, C. P., 2016. Fitting the Michaelis-Menten model. Journal of Computational and Applied Mathematics 296, 303-319.

Vanlier, J., Tiemann, C. A., Hilbers, P. A., van Riel, N. A., 2012. A bayesian approach to targeted experiment design. Bioinformatics 28 (8), 1136-1142.

Von Stosch, M., Oliveira, R., Peres, J., de Azevedo, S. F., 2014. Hybrid semi-parametric modeling in process systems engineering: Past, present and future. Computers \& Chemical Engineering 60, 86-101.

Wang, M., Jacobson, W. E., Chotteau, V., Hjalmarsson, H., Jacobsen, E., Chotteau, V., Hjalmarsson, H., 2019. A Multi-Step Least-Squares Method for Nonlinear Rational Models. In: Proc. 2019 American Control Conference (ACC). IEEE, Philadelphia, PA.

Wouwer, A. V., Renotte, C., Bogaerts, P., 2004. Biological reaction modeling using radial basis function networks. Computers \& chemical engineering 28 (11), 2157-2164.

Zou, H., 2006. The adaptive lasso and its oracle properties. Journal of the American statistical association 101 (476), 1418-1429. 